\title{
How stable is lung function in patients with stable chronic obstructive pulmonary disease when monitored using a telehealth system? A longitudinal and home-based study
}

Åsa Holmner ${ }^{1,2}$, Fredrik Öhberg ${ }^{1}$, Urban Wiklund ${ }^{1}$, Eva Bergmann ${ }^{3}$, Anders Blomberg ${ }^{3}$ and Karin Wadell ${ }^{3,4^{*}}$ (D)

\begin{abstract}
Background: Many telehealth systems have been designed to identify signs of exacerbations in patients with chronic obstructive pulmonary disease (COPD), but few previous studies have reported the nature of recorded lung function data and what variations to expect in this group of individuals. The aim of the study was to evaluate the nature of individual diurnal, day-to-day and long-term variation in important prognostic markers of COPD exacerbations by employing a telehealth system developed in-house.

Methods: Eight women and five men with COPD performed measurements (spirometry, pulse oximetry and the COPD assessment test (CAT)) three times per week for 4-6 months using the telehealth system. Short-term and long-term individual variations were assessed using the relative density and weekly means respectively. Quality of the spirometry measurements (forced expiratory volume in one second $\left(\mathrm{FEV}_{1}\right)$ and inspiratory capacity (IC)) was assessed employing the criteria of American Thoracic Society (ATS)/European Respiratory Society (ERS) guidelines.

Results: Close to 1100 measurements of both $\mathrm{FEV}_{1}$ and IC were performed during a total of 240 patient weeks. The two standard deviation ranges for intra-individual short-term variation were approximately $\pm 210 \mathrm{~mL}$ and $\pm 350 \mathrm{~mL}$ for FEV and IC respectively. In long-term, spirometry values increased and decreased without notable changes in symptoms as reported by CAT, although it was unusual with a decrease of more than $50 \mathrm{~mL}$ per measurement of $\mathrm{FEV}_{1}$ between three consecutive measurement days. No exacerbation occurred. There was a moderate to strong positive correlation between $\mathrm{FEV}_{1}$ and IC, but weak or absent correlation with the other prognostic markers in the majority of the participants.

Conclusions: Although FEV 1 and IC varied within a noticeable range, no corresponding change in symptoms occurred. Therefore, this study reveals important and, to our knowledge, previously not reported information about short and longterm variability in prognostic markers in stable patients with COPD. The present data are of significance when defining criteria for detecting exacerbations using telehealth strategies.
\end{abstract}

Keywords: COPD, Spirometry, Home-monitoring, Long-term variations, Exacerbations, Telehealth

* Correspondence: karin.wadell@umu.se

${ }^{3}$ Department of Public Health and Clinical Medicine, Section of Medicine, Umeå University, Umeå, Sweden

${ }^{4}$ Department of Community Medicine and Rehabilitation, Physiotherapy, Umeå University, Umeå, Sweden

Full list of author information is available at the end of the article

(c) The Author(s). 2020 Open Access This article is licensed under a Creative Commons Attribution 4.0 International License, which permits use, sharing, adaptation, distribution and reproduction in any medium or format, as long as you give appropriate credit to the original author(s) and the source, provide a link to the Creative Commons licence, and indicate if changes were made. The images or other third party material in this article are included in the article's Creative Commons licence, unless indicated otherwise in a credit line to the material. If material is not included in the article's Creative Commons licence and your intended use is not permitted by statutory regulation or exceeds the permitted use, you will need to obtain permission directly from the copyright holder. To view a copy of this licence, visit http://creativecommons.org/licenses/by/4.0/ The Creative Commons Public Domain Dedication waiver (http://creativecommons.org/publicdomain/zero/1.0/) applies to the data made available in this article, unless otherwise stated in a credit line to the data. 


\section{Background}

Chronic obstructive pulmonary disease (COPD) is one of the most prevalent, costly and deadly non-communicable disease $[1,2]$ and the importance of effective COPD management has received a lot of attention. Spirometry (i.e. forced expiratory volume in one second $\left(\mathrm{FEV}_{1}\right)$ and forced vital capacity (FVC)) is used for diagnosis and disease monitoring and these parameters are, by definition, seen as stable as long as the patients are in a stable state of the disease [3]. A major cause of hospitalization in COPD is acute exacerbations (AE), defined as a worsening of the patient's respiratory symptoms that is beyond normal dayto-day variation [3]. AEs contribute significantly to healthcare consumption, hence the costs for COPD care, and patients with two or more exacerbations per year have a poor prognostic outcome [3, 4]. Regular monitoring of vital signs, physiological parameters or symptoms using telemonitoring or telehealth $(\mathrm{TH})$ approaches has been proposed as a strategy for supporting early detection of exacerbations, allowing prompt initiation of treatment and faster recovery [5-7]. However, to be able to use these monitored physiological parameters as prognostic markers it is important to be aware of their inherent short and long-term variation, and their relation to clinically relevant symptoms, when recorded in a real-life situation.

Whilst the existing evidence is ambiguous, several $\mathrm{TH}$ studies have reported positive outcomes on hospital admissions and exacerbation frequency in COPD [8,9]. Other studies report improved symptom control, functional status [10], self-management capacity [11] and health-related quality of life [12] compared to controls. There are also studies that have gone further and identified promising markers of COPD exacerbations [13-15], although some demonstrate poor predictive performance or even poor clinical outcomes [16-18]. Other studies have failed to draw any conclusions at all on the predictive performance of monitored parameters but, nonetheless, generated a positive outcome in terms of a reduction in exacerbation frequency [19]. A recent review including 29 telemonitoring studies on COPD, confirm these conflicting results with an almost equal distribution of improvements and no improvements in patient outcomes in the studies [20]. Common to most studies, however, is that they fail to report the nature of recorded data, e.g. with respect to differences within and between individuals, diurnal or day-to-day variations, frequency of outliers, long-term stability or variability and individual differences in adherence or learning effects. These data are needed to be able to judge the feasibility of a $\mathrm{TH}$ approach and to identify individuals suitable for enrolment in such a program. As it is well known that COPD is a heterogeneous disease, it is critical to take the individual at the starting point when designing $\mathrm{TH}$ programs and interventions. This knowledge-gap is the point of departure of the present study.
The aim of the study was to evaluate the nature of individual diurnal, day-to-day and long-term variation in spirometry measurements $\left(\mathrm{FEV}_{1}\right.$ and inspiratory capacity (IC)), and their co-variation with other important prognostic markers of COPD exacerbations (oxygen saturation $\left(\mathrm{SpO}_{2}\right)$ and symptoms measured with $\mathrm{COPD}$ assessment test (CAT)) by employing a telehealth system developed in-house.

\section{Methods \\ Mobile home monitoring system}

Data collection was enabled through a mobile health monitoring system developed in-house and described elsewhere [21]. In summary, the system consisted of a spirometer (SpiroTube Mobile Edition, Thor Laboratories, Hungary) connected via Bluetooth or cable to a tablet computer (Acer Iconia Tab W501P, 2011), providing instructions for spirometry manoeuvres and an electronic version of the CAT [22]. To permit recording saturation, another potential prognostic marker, the system was upgraded prior to the present study with an application for a Bluetooth-connected pulse oximeter (Nonin WristOx model 3250, Nonin Medical Inc., Plymouth, $\mathrm{MN})$. The final version of the system thus consisted of a tablet computer with three applications, one for the $\mathrm{CAT}$, one for pulse oximetry and one for a spirometry protocol. Unprocessed data were stored locally until a mobile broadband connection was established and thereafter automatically uploaded to a secure server for further processing.

\section{Participants and study design}

During the years 2013-2015, consecutive participants with moderate to very severe COPD according to Global initiative for chronic Obstructive lung disease (GOLD) guidelines [3] were recruited from the Department of Medicine, Division of Respiratory Medicine and Allergy, University hospital of Umeå, Sweden. Care was taken to include patients with a history of exacerbations. After obtaining informed consent, participants completed an outpatient visit that included standard medical examination, chest $\mathrm{x}$-ray, static and dynamic spirometry, venous blood tests and six-minute walk tests [23]. Baseline value of the participant's perceived respiratory impairment was obtained using the modified Medical Research Council (mMRC) dyspnoea scale [24].

The study had a pragmatic approach and provided a minimum of individual education prior to enrolment as well as limited support during the trial. Before returning home with the equipment, participants received a short instruction (15-20 $\mathrm{min}$ ) to the home monitoring system and were asked to execute the study protocol with guidance from a researcher involved in the study. The protocol implemented on the tablet computer was executed 
in the following order: First, the participant was asked to answer the eight questions of the CAT. This test was followed by pulse oximetry for $1 \mathrm{~min}$. Finally, the participant executed a spirometry protocol consisting of a minimum of three IC manoeuvres, followed by a minimum of three $\mathrm{FEV}_{6}$ manoeuvres to enable calculation of $\mathrm{FEV}_{1}$. It was possible to repeat a manoeuvre if needed. During spirometry manoeuvres, participants were instructed to sit in an upright position with a 90-degree flexion in hips and knees and to use a nose clip.

The first week of the study, the participants performed the above-mentioned protocol twice a day. Thereafter, the protocol was repeated morning and evening 3 days a week for 4-6 months. The frequency of 3 days per week was chosen to closely monitor potential variation, yet achieve a relevant compliance to the procedure. Participants inhaled subscribed medications before the morning spirometry procedures. When prescribed inhalation therapy bi daily, the evening spirometry was carried out before drug administration. Technical support was provided during the study, but the participants did not receive any medical follow-up based on the recorded data. Participants were thus aware that they should contact their usual healthcare provider if they needed medical attention.

After completion of the study, the participants performed another outpatient visit, including one 6-min walk test, dynamic spirometry and assessment of perceived respiratory impairment (mMRC).

\section{Data management \& statistical analysis}

During ongoing measurements, oxygen saturation, heart rate and CAT scores were displayed to the participants. During the $\mathrm{FEV}_{1}$ and IC manoeuvres, visual cues indicating airflow direction were shown to the user, but no results from the spirometry were presented on the device. After the recording, data were uploaded to a server at the hospital for further analyses. More details regarding the recording and processing of the recorded lung function signals can be found in the Supplementary Materials. All data processing and statistics were performed using MATLAB (R2016b, MathWorks, Natick, Massachusetts, USA) and R software (version 3.3.3).

Feasibility of home spirometry was judged by estimating the overall adherence to the study protocol and based on the quality of the $\mathrm{FEV}_{1}$ and IC measurements, as defined by the American Thoracic Society (ATS)/ European Respiratory Society (ERS) guidelines [25]. Adherence to the study protocol was defined as the ratio between the number of times the spirometry protocol was executed and the total number of scheduled measurements for the whole study period, i.e., six times a week. The first 2 weeks of the study were classified as a learning phase and thus excluded from the analysis, as were shorter interruptions owing to breaks that were agreed upon or unforeseen system maintenance.

The quality of each breathing manoeuvre was visually inspected by one investigator. Occasionally, patients had performed more than three efforts to obtain three tests that they considered acceptable, but the three best measurements were selected for calculation of $\mathrm{FEV}_{1}$ and IC, according to the ATS/ERS guidelines [25].

The adherence to the ATS/ERS guidelines was evaluated by calculating the difference between the largest and second largest $\mathrm{FEV}_{1}$. If the difference was $<0.150 \mathrm{~L}$, the test was considered acceptable. The difference was also compared with the threshold $0.100 \mathrm{~L}$, which is the corresponding threshold for participants with a FVC below 1.0 L. In this study, all participants presented with FVC $>1.0 \mathrm{~L}$ at the baseline post-bronchodilator examination. Pearson's correlation coefficient was calculated to assess the agreement between the different measured recordings.

For IC, the coefficient of variation (CV) was determined between the three acceptable manoeuvres. Since the mean $\mathrm{CV}$ in chronic airflow obstruction has been found to be approximately $5 \%$ [26], the quality of the IC data was evaluated by comparing $\mathrm{CV}$ with the thresholds 5,8 and $10 \%$, respectively.

The largest $\mathrm{FEV}_{1}$ and the average IC from each test occasion were used in further analyses. All recordings (heart rate, $\mathrm{SpO}_{2}, \mathrm{FEV}_{1}, \mathrm{IC}$ and $\mathrm{CAT}$ ) were thereafter compiled and analysed on group level as well as on individual level with respect to the individual diurnal, dayto-day and long-term variations.

An analysis of factors affecting the different outcome measures on a group level was performed using linear mixed models (R-package 'Ime4' version 1.1.12), where the response measure was set to $\mathrm{FEV}_{1}, \mathrm{IC}, \mathrm{SpO}_{2}$ and CAT. The model included the fixed effects of sex (male and female), measurement period (beginning, mid and late) and time of day: morning (AM) and afternoon/ evening (PM). The included random effect was a random intercept on participant identity.

The long-term variation was assessed by calculating the mean of all measurements in the same week. The short-term variation in each participant, including the diurnal variation, was evaluated by estimating the relative density of changes between successive measurements and between measurement performed at the same time of the day (morning or evening). Additionally, the presence of periods with successive decreases between measurements was determined by defining a significant change as a decrease $>50 \mathrm{ml}$ in $\mathrm{FEV}_{1}$ or IC between two consecutive measurements. Thus, the total decrease was $>100 \mathrm{ml}$ between the first and third of the successive measurements. We only considered changes between measurements from the same part of the day, i.e., from 
morning-to-morning or evening-to-evening, respectively. In addition, if a period with successive decreases over 2 days or more was found, $\mathrm{FEV}_{1}$ or IC was considered as unchanged if the next value increased with less than 50 $\mathrm{ml}$. As soon as the next value increased $>50 \mathrm{ml}$ from the previous value, the period with successive decreases was considered to have ended.

\section{Results}

\section{Study population}

Twenty-five participants fulfilling the inclusion criteria were asked for participation during the two-year inclusion period. Thirteen accepted to take part, and were included in the study. The recruited study population consisted of a heterogeneous group of participants represented by eight women and five men aged 48 to 79 years. The participants had a BMI ranging from 15.733.7, a $\mathrm{FEV}_{1} \%$ predicted ranging from 21 to 68 and $\mathrm{SpO}_{2}$ at rest ranging from 90 to $100 \%$. The thirteen participants were spirometrically graded as GOLD $2 n=6$; GOLD 3, $n=5$; and GOLD 4, $n=2$. Twelve participants were characterized as GOLD stage D and one participant as GOLD stage A. Eight of the thirteen participants had a history of exacerbations in the preceding 12 months and five of them had had more than one. All but one remained stable throughout the study and the only confirmed exacerbation happened during a period when the system was on maintenance; thus, it could not be accounted for in the analysis. Two participants (GOLD stage 3D) were excluded from all analyses because of technical failure of the device. This resulted in a loss of nearly all recorded data in one participant, and very few performed recordings in the other participant. Characteristics of the $11 \mathrm{in}$ cluded patients are presented in Table 1.

\section{Adherence to study protocol and quality of home spirometry}

After the initial learning period of 14 days, the remaining 11 participants recorded a total of 1090 forced expiratory volume measurements and 1088 inspiratory capacity measurements that were used to calculate $\mathrm{FEV}_{1}$ and IC according to the ERS/ATS guidelines [25]. The average adherence to the study protocol was $90.6 \%$, defined as the ratio between the total number of times the spirometry procedure was executed and the total number of scheduled procedures. That is, less than one in ten recordings was skipped or forgotten (reasons for not performing manoeuvres are unknown).

A complete set of three correct $\mathrm{FEV}_{1}$ manoeuvres was recorded on 1070 occasions (98.2\%) when the spirometry protocol was executed. On 16 occasions (1.5\%), two correct procedures were recorded and on four additional occasions only one correct manoeuvre was recorded $(<$ $1 \%)$. The between-manoeuvre variability, based on the
Table 1 Baseline characteristics of study subjects

\begin{tabular}{|c|c|c|}
\hline \multirow[t]{2}{*}{$\operatorname{Sex}(f / m) 8 / 3$} & \multicolumn{2}{|l|}{ Baseline } \\
\hline & Median & IQR \\
\hline Age & 67 & $59-72$ \\
\hline Height (m) & 1.66 & $1.59-1.76$ \\
\hline Weight (kg) & 65 & $59-87$ \\
\hline BMI & 26 & $21-30$ \\
\hline VC (L) & 2.70 & $2.6-3.9$ \\
\hline VC (\% pred) & 112 & 89-119 \\
\hline FVC (L) & 2.15 & $1.8-3.0$ \\
\hline FVC (\% pred) & 80 & $75-82$ \\
\hline $\mathrm{FEV}_{1}(\mathrm{~L})$ & 1.01 & $0.9-1.7$ \\
\hline $\mathrm{FEV}_{1}(\%$ pred $)$ & 53 & $36-63$ \\
\hline FEV\% & 54 & $38-62$ \\
\hline IC (L) & 1.89 & $1.6-2.5$ \\
\hline IC (\% pred) & 98 & $82-106$ \\
\hline $6 M W D(m)^{a}$ & 387 & $335-479$ \\
\hline $\mathrm{SpO}_{2}$ rest & 96 & $95-98$ \\
\hline mMRC & 2 & $1.5-4$ \\
\hline
\end{tabular}

BMI Body Mass Index, VC Vital Capacity, FVC Forced Vital Capacity, FEV, Forced Expiratory Volume in one second, $I C$ Inspiratory Capacity, $6 M W T 6$ min walking test, $\mathrm{SpO}_{2}$ Oxygen saturation measured by pulse oximetry, $m M R C$ modified Medical Research Council Dyspnea Scale, IQR Inter Quartile Range (25-75), pred predicted, ${ }^{a}$ best of two tests

difference between the highest and second highest FEV value, was calculated for all occasions with at least two acceptable recordings and resulted in $\leq 150 \mathrm{ml}$ on $97.7 \%$ occasions and $\leq 100 \mathrm{ml}$ on $93.1 \%$ occasions.

Of the 1088 occasions when the IC manoeuvre was performed, three correct manoeuvres were performed on 1071 occasions (98.3\%), enabling calculation of an IC value. On 16 occasions (1.5\%), only two correct manoeuvres were recorded and on one additional occasion only one correct manoeuvre was recorded. The between manoeuvre variability, estimated by the coefficient of variation $(\mathrm{CV})$ on all occasions with a minimum of two acceptable measurements, resulted in a $\mathrm{CV} \leq 10 \%$ in $88.2 \%$ of the occasions, a $\mathrm{CV} \leq 8 \%$ in $79.0 \%$ of the occasions and a $\mathrm{CV} \leq 5 \%$ in $56.2 \%$, i.e., slightly more than half of the occasions.

\section{Home vs lab measurements}

After the initial learning period, spirometry, pulse oximetry and CAT scoring were executed twice daily, morning and evening, three times a week. Table 2 and Table 3 present spirometry data from the first and last months compared to the participants' baseline values. $\mathrm{FEV}_{1}$ and IC from home monitoring are presented as the ratio between the value from home monitoring and the baseline value. The unsupervised measurements generally produced lower values of $\mathrm{FEV}_{1}$, although there 
Table 2 Monthly averages of FEV 1 compared to measurements at the baseline examination

\begin{tabular}{|c|c|c|c|c|c|c|}
\hline \multirow[t]{2}{*}{ Subject } & \multirow{2}{*}{$\begin{array}{l}\text { Predicted } \\
\text { value }(L)\end{array}$} & \multirow[t]{2}{*}{ Baseline (L) } & \multicolumn{2}{|c|}{ Home ${ }^{a}$ - First month } & \multicolumn{2}{|c|}{ Home $^{\mathrm{a}}$ - Last month } \\
\hline & & & $\mathrm{AM}$ & PM & $A M$ & PM \\
\hline 1 & 1.70 & 0.85 & 0.82 & 0.79 & 0.92 & 0.85 \\
\hline 2 & 3.48 & 1.01 & 0.54 & 0.77 & 0.58 & 0.77 \\
\hline 3 & 2.43 & 0.85 & 0.97 & 0.99 & 0.99 & 1.04 \\
\hline 4 & 1.79 & 0.95 & 0.84 & 0.91 & 0.75 & 0.84 \\
\hline 5 & 1.89 & 0.7 & 1.03 & 0.83 & 1.10 & 0.84 \\
\hline 6 & 1.95 & 1.21 & 0.81 & 0.79 & 0.83 & 0.78 \\
\hline 7 & 2.89 & 1.76 & 0.86 & 0.90 & 0.87 & 0.90 \\
\hline 8 & 2.57 & 0.54 & 0.89 & 0.99 & 0.84 & 0.97 \\
\hline 9 & 3.00 & 2.04 & 0.97 & 0.89 & 0.81 & 0.79 \\
\hline 10 & 2.55 & 1.68 & 0.66 & 0.63 & 0.67 & 0.65 \\
\hline 11 & 3.25 & 2.05 & 0.88 & 0.77 & 0.76 & 0.77 \\
\hline Mean (SD) & $2.50(0.61)$ & $1.24(0.55)$ & $0.84(0.14)$ & $0.84(0.11)$ & $0.83(0.14)$ & $0.84(0.11)$ \\
\hline
\end{tabular}

were participants that managed to pace themselves well enough to match the lab results (e.g., six participants with $\mathrm{FEV}_{1}$ (home) $/ \mathrm{FEV}_{1}$ (baseline) $\geq 0.9$ in $\mathrm{AM}$ and/or PM measurements during the first month). Unsupervised IC values were generally lower than the lab values. A notable difference $(>100 \mathrm{ml}$ ) between morning and evening values were found in several participants in both $\mathrm{FEV}_{1}$ and IC during both the first and last month: e.g. in FEV 1 from the first month in participants 5 and 11 . The nature of this difference was, however, not consistent; some participants systematically recorded higher lung function values in the morning, whereas others reached higher values in the afternoon.

As shown in Table 4, there was a moderate to strong positive correlation between $\mathrm{FEV}_{1}$ and IC in 9 of 11 subjects. However, the correlation between spirometry measurements and the other two prognostic COPD markers were in general absent or weak, except that the last four included subjects presented with a moderate to strong negative correlation between IC and CAT.

\section{Diurnal, day-to-day and long-term variations in lung function}

To evaluate the variability in the recordings on shorter timescales, which is of relevance for disease management and telehealth purposes, we calculated the intraindividual variation in $\mathrm{FEV}_{1}$ and IC between successive measurements and between weeks.

Figure 1 presents the distribution of weekly means in $\mathrm{FEV}_{1}$ (\% predicted), IC (\% predicted), $\mathrm{SpO}_{2}$, and CAT for all participants, overlaid on the corresponding lab

Table 3 Monthly averages of IC compared to measurements at the baseline examination

\begin{tabular}{|c|c|c|c|c|c|c|}
\hline \multirow[t]{2}{*}{ Subject } & \multirow{2}{*}{$\begin{array}{l}\text { Predicted } \\
\text { value }(\mathrm{L})\end{array}$} & \multirow[t]{2}{*}{ Baseline (L) } & \multicolumn{2}{|c|}{ Home $^{\mathbf{a}}$ - First month } & \multicolumn{2}{|c|}{ Home $^{\mathbf{a}}$ - Last month } \\
\hline & & & AM & PM & AM & PM \\
\hline 1 & 1.67 & 1.64 & 0.87 & 0.88 & 0.84 & 0.83 \\
\hline 2 & 2.58 & 1.65 & 0.70 & 0.90 & 0.74 & 0.96 \\
\hline 3 & 2.03 & 1.58 & 0.83 & 0.82 & 0.87 & 0.91 \\
\hline 4 & 1.77 & 1.89 & 0.81 & 0.83 & 0.75 & 0.83 \\
\hline 5 & 1.52 & 1.47 & 0.84 & 0.76 & 0.94 & 0.81 \\
\hline 6 & 1.78 & 1.37 & 0.79 & 0.80 & 0.87 & 0.87 \\
\hline 7 & 2.58 & 2.22 & 0.58 & 0.62 & 0.79 & 0.81 \\
\hline 8 & 2.35 & 2.33 & 0.74 & 0.77 & 0.78 & 0.83 \\
\hline 9 & 3.05 & 3.29 & 0.65 & 0.63 & 0.58 & 0.57 \\
\hline 10 & 2.50 & 2.62 & 0.72 & 0.68 & 0.65 & 0.60 \\
\hline 11 & 3.34 & 3.57 & 0.94 & 0.90 & 0.98 & 0.95 \\
\hline Mean (SD) & $2.29(0.59)$ & $2.15(0.74)$ & $0.77(0.11)$ & $0.78(0.10)$ & $0.80(0.12)$ & $0.82(0.13)$ \\
\hline
\end{tabular}

Values of home measurements are expressed as the ratio Home/Baseline $\left(^{\mathrm{a}}\right)$, where Home is the monthly average of AM and PM measurements, respectively 
Table 4 Correlation between prognostic markers of COPD exacerbations (spirometry, CAT and $\mathrm{SpO}_{2}$ )

\begin{tabular}{llllll}
\hline Subject & FEV $\mathbf{1}$ vs IC & IC vs CAT & FEV $\mathbf{1}$ vs CAT & $I_{\text {V v SpO }}$ & $F_{\mathbf{2}}$ \\
\hline 1 & 0.48 & -0.04 & -0.14 & 0.22 & 0.20 \\
2 & 0.90 & -0.15 & -0.20 & 0.14 & 0.15 \\
3 & 0.45 & -0.06 & -0.01 & 0.03 & 0.04 \\
4 & 0.60 & -0.19 & -0.18 & -0.01 & 0.07 \\
5 & 0.66 & 0.03 & 0.08 & -0.16 & 0.07 \\
6 & 0.44 & -0.16 & -0.19 & -0.01 & 0.10 \\
7 & -0.03 & -0.13 & 0.13 & -0.19 & 0.09 \\
8 & 0.42 & -0.35 & 0.12 & 0.20 & 0.31 \\
9 & 0.53 & -0.47 & -0.41 & -0.03 & -0.06 \\
10 & 0.58 & -0.42 & -0.15 & -0.03 & -0.28 \\
11 & 0.29 & -0.58 & 0.18 & 0.21 & -0.08 \\
Median (IQR) & $0.48(0.17)$ & $-0.16(0.32)$ & $-0.14(0.30)$ & $-0.01(0.22)$ & $0.07(0.17)$ \\
\hline
\end{tabular}

Values are correlation coefficients. FEV ${ }_{1}$ Forced Expiratory Volume in one second, IC Inspiratory Capacity, CAT COPD Assessment Test, $\mathrm{SpO}_{2} \mathrm{Oxygen}$ saturation measured by pulse oximetry, IQR Inter Quartile Range

values that were obtained at the time of enrolment in the study (see Table 1).

Figure 2 shows the variation between consecutive measurements, which typically have a frequency of three weekly measurements (2-3 days between each measurement). The variability is presented using estimated density functions based on between-measurements changes in $\mathrm{FEV}_{1}$ and $\mathrm{IC}$ (percent of predicted values). There were marked differences in between-measurements variation between the participants, but also differences between individuals with respect to diurnal variation across several days. The variability between consecutive measurements (Fig. 2, top panels) showed two participants with a low and wide distribution due to a marked diurnal variation in $\mathrm{IC}$, but distributions with two peaks also indicated a notable diurnal variation. Therefore, the distribution of consecutive AM-AM (middle panels) and PM-PM (bottom panels) are also presented in Fig. 2.
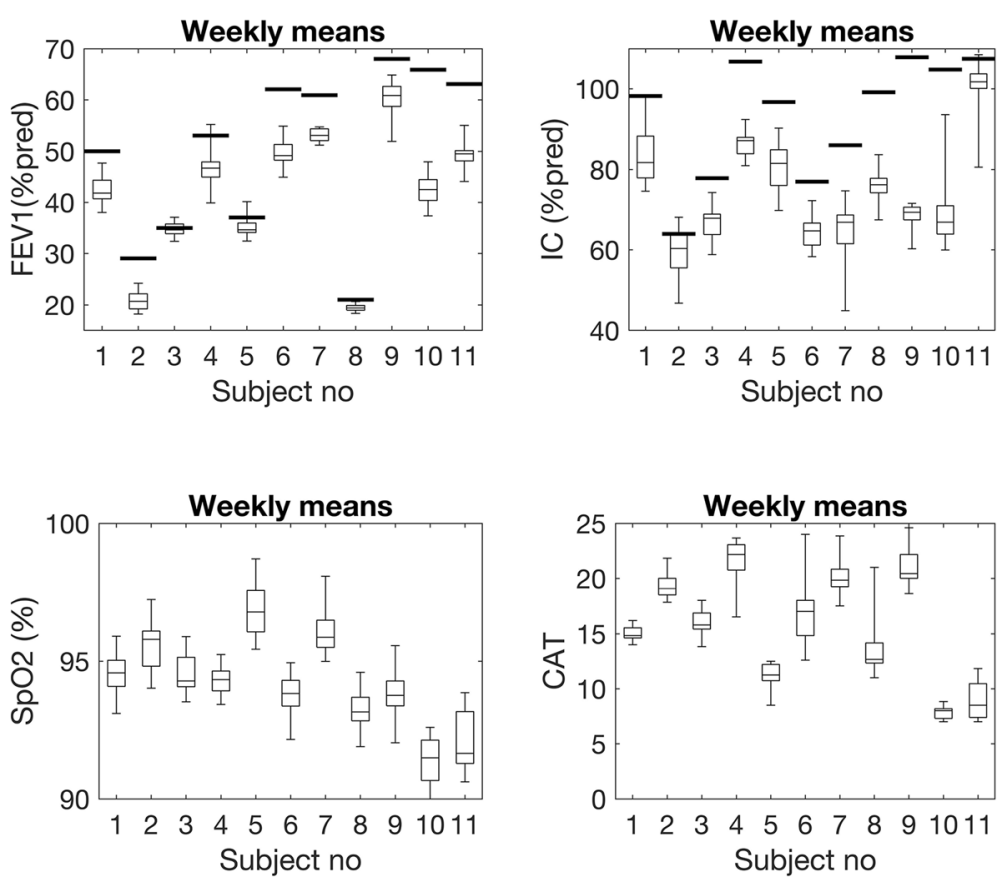

Fig. 1 Weekly means of $\mathrm{FEV}_{1}$ (\% of predicted), IC (\% of predicted), $\mathrm{SpO}_{2}$ and CAT. Boxes show median and IQR, whiskers show range. Solid lines show the corresponding measurements from the baseline spirometry before the start of home monitoring. $\mathrm{FEV}_{\mathbf{1}}$ : Forced Expiratory Volume in one second, IC: Inspiratory Capacity, $\mathrm{SpO}_{2}$ : Oxygen saturation measured by pulse oximetry, CAT: COPD Assessment Test 

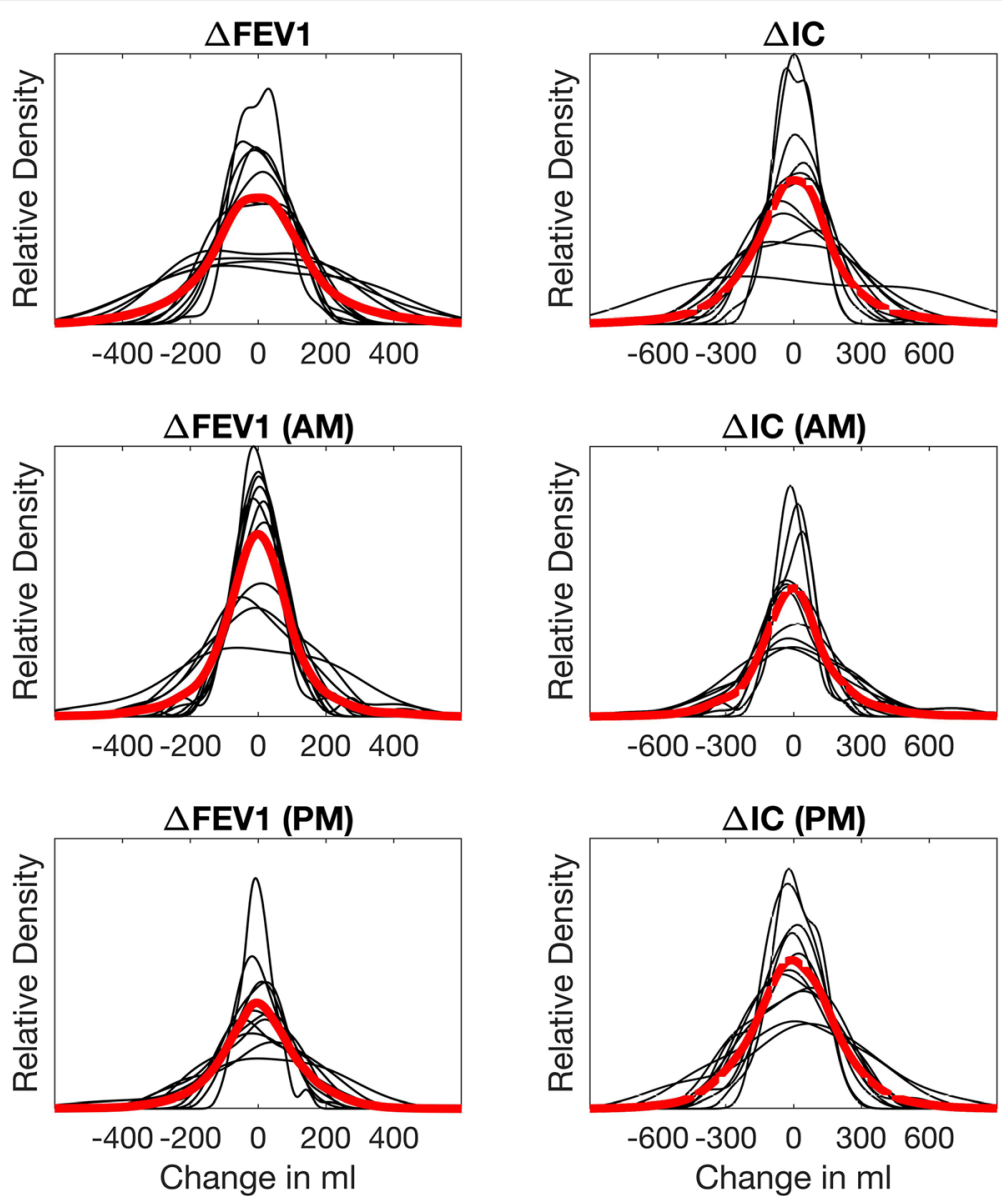

Fig. 2 Relative density of the changes in of $\mathrm{FEV}_{1}$ (left) and IC (right) between measurements (top), between successive AM measurements (middle), and between successive PM measurements (bottom). Thin lines show individual curves. Red thick lines show the averaged density curve. FEV $\mathbf{1}_{\mathbf{1}}$ : Forced Expiratory Volume in one second, IC: Inspiratory Capacity

The 2-SD range was -211 to $210 \mathrm{ml}$ for $\Delta \mathrm{FEV}_{1}(\mathrm{AM})$ ( -8 to $8 \%$ for $\triangle \mathrm{FEV}_{1}$ in \%pred) and -209 to $213 \mathrm{ml}$ for $\triangle \mathrm{FEV}_{1}(\mathrm{PM})\left(-9\right.$ to $8 \%$ for $\triangle \mathrm{FEV}_{1}$ in \%pred), respectively. For IC, the 2-SD range was higher: -286 to $351 \mathrm{ml}$ for $\triangle \mathrm{IC}$ (AM) (-13 to $14 \%$ for $\Delta \mathrm{IC}$ in \%pred) and -316 to $384 \mathrm{ml}$ for $\Delta \mathrm{IC}(\mathrm{PM})(-15$ to $15 \%$ for $\Delta \mathrm{IC}$ in \% pred), respectively.

When comparing measurements performed at the same time of the day, the analysis of successive decreases revealed a total of 34 periods in the 11 patients where $\mathrm{FEV}_{1}$ decreased with a total of $>50 \mathrm{ml}$ over several consecutive measurements: 30 two-day periods and 4 threeday periods. After 29 of these 34 periods, $\mathrm{FEV}_{1}$ increased $>50 \mathrm{ml}$, whereas it remained unchanged 5 times. Moreover, in $30 / 34$ of these periods, $\mathrm{FEV}_{1}$ was higher than the participant's overall mean value before the onset of the decrease. There were only six two-day periods where $\mathrm{FEV}_{1}$ decreased with a total of $>100 \mathrm{ml}$ over several consecutive measurements; in all these periods, $\mathrm{FEV}_{1}$ was higher than the overall mean value before the onset of the decrease. The same analysis for IC showed a total of
62 periods with successive decreases of $>50 \mathrm{ml}$ from measurement-to-measurement: 51 two-day sequences and 11 three-day sequences. Of those, in 55/62 sequences (including all three-day periods), IC was higher than the mean before the onset of the decrease. There were 26 two-day periods and one three-day period where IC decreased with a total of $>100 \mathrm{ml}$ over consecutive measurements; before the onset of the decrease IC was higher than the overall mean value in 23 of these periods.

On a group level, the linear mixed model analysis showed that $\mathrm{FEV}_{1}$ was significantly smaller during the middle period $(-0.02 \mathrm{~L})$ compared to baseline. By the end of the measurement period, that difference has disappeared. In contrast, IC differed both in the middle and the end of the measurement period compared to baseline. In both cases, IC was $0.06 \mathrm{~L}$ higher compared to the baseline level. Also, there was a difference between AM and PM measures, where the measurements were $0.04 \mathrm{~L}$ higher in PM compared to AM. For $\mathrm{SpO}_{2}$, there was only a negligible difference $(0.4 \%$ lower $)$ in the final 
phase compared to baseline For CAT, the estimated values were lower; -0.99 units and -0.73 units below the middle and final stage respectively, compared with baseline.

\section{Discussion}

As one of the first, this study reports important information about habitual variability in lung function, oxygen saturation and disease-related symptoms in individuals with COPD. The findings are based on more than one thousand unsupervised $\mathrm{FEV}_{1}$ and IC-recordings collected in a home environment. The study was originally designed to investigate whether a newly developed home monitoring system could detect early signs of exacerbation. Thus, care was taken to recruit patients with a recent history of exacerbations (10 out of 11 patients were GOLD D). Despite this focused selection, only one participant exacerbated during the whole study period. The reason for this is unclear, but might be a consequence of enhanced disease awareness, increased attention from health care, that the participants performed a set of breathing exercises regularly, or a combination of any of the above. This outcome per se is not unique, several telehealth studies have reported a reduction in exacerbation frequency and hospital admissions in telehealth groups compared to controls $[9,19,27]$, which is one of the arguments for employing telehealth at all. For example, Segrelles-Calvo et al. demonstrated that monitoring of lung function, $\mathrm{SpO}_{2}$, heart rate and blood pressure with a $\mathrm{TH}$ system in patients on long-term home oxygen therapy significantly reduced the number of emergency visits, hospital admissions, length of hospital stay and need for non-invasive mechanical ventilation, compared to controls receiving conventional care [9]. These are all cost driving interventions suggesting a cost-benefit of the intervention. Tupper et al. also found improvements in health-related quality of life in patients equipped with a $\mathrm{TH}$ system recording symptoms, $\mathrm{SpO}_{2}$ and spirometry in addition to video consultations [12]. Unfortunately, there is very little information available on the nature of the telehealth data recorded in these studies and we have chosen to report data on short and long-term variations in measured indices; the inter and intra-measurement variability between measurements and adherence to the protocol. Moreover, we have chosen to report data also on an individual level, such as differences in diurnal and day to day variations in performance, and to highlight factors that are relevant to take into consideration when selecting suitable patients for employment of a TH system in ordinary care.

First, we estimated how well the 11 participants managed to perform the unsupervised spirometry by classifying all recorded spirometry manoeuvres either as correctly or incorrectly performed, as defined by the
ATS/ERS guidelines [25]. Thereafter, the measurements were assessed with respect to repeatability, i.e., the within manoeuvre variability each time the spirometry protocol was executed, and with respect to adherence to the study protocol. Based on this assessment, we conclude that spirometry is indeed feasible to employ in a home environment. More than $98 \%$ of the occasions produced three acceptable manoeuvres with acceptable within manoeuvre variability, and adherence to the study protocol was judged to be very high, as more than $98 \%$ of scheduled routines were performed as planned. Compared to the pre-post $\mathrm{FEV}_{1}$ measurements, the selfpaced measurements were typically lower although some managed to perform nearly as well at home as in the lab. Nonetheless, although slightly lower, the measurements at home remained stable and varied very little, particularly when comparing measurements performed the same time of the day over time. The same pattern was found in a study by Rodriguez-Roisin et al., who investigated change over time as a part of the WISDOM (Withdrawal of Inhaled Steroids During Optimized Bronchodilator Management) trial by having patients with severe to very severe COPD performing spirometry at home. Whilst they found a small difference with lower $\mathrm{FEV}_{1}$ values recorded at home compared with in the clinic, the change remained consistent and the data confirmed good agreement between measurements at home and at the clinic [28].

We did find cases in which $\mathrm{FEV}_{1}$ decreased during several consecutive measurements but without any signs of exacerbations or change in symptoms. A total of 34 periods were found where $\mathrm{FEV}_{1}$ decreased more than $100 \mathrm{ml}$ between 3 and 4 consecutive measurements in total. A majority of those returned towards the "pre-decrease" values at the next measurement. Janssens et al. suggest that a change greater than $0.280-0.320 \mathrm{~L}$ is required to exceed the normal between-test variation [29]. However, the ATS/ERS guidelines report a minimal clinical important difference for $\mathrm{FEV}_{1}$ of $100 \mathrm{ml}$ [30]. In addition, Redelmeier et al. state that changes within the normal variation may still represent a clinically meaningful result when accompanied by symptom changes [31].

Despite the lack of long-term trends in lung function, as indicated by the variation in weekly means of $\mathrm{FEV}_{1} \%$ predicted for each participant (Fig. 1), there is an evident difference in variability in spirometry both with respect to the variability between successive measurement and how the weekly means vary over time. The latter implies that a lung function value deviating from that of the previous week +/- the SD can still be within the normal range and does not necessarily imply a relevant change in lung function or an upcoming exacerbation, especially without corresponding change in symptoms, which was the case in this study. 
Despite this variability in lung function measurements, we have drawn the conclusion that home spirometry is indeed feasible, though it should not be compared with measurements performed under supervision. The lung function values in the present study were consistently lower when performed at home compared to in clinic except for a few participants. Noted variations are thus not necessarily owing to poor spirometry performance. Compared to the prepost values, the participants stayed consistent in their measurement results over time, though at a lower value (Fig. 1). None of the differences we found in the linear mixed model (on group level) were of clinical relevance. Another argument supporting this feasibility statement is that three complete and acceptable manoeuvres were performed in about $98 \%$ of the cases. In the UPLIFT study where the patients were followed with spirometry performed at clinic every sixth month for 4 years [29], they found that $86-92 \%$ of the manoeuvres were correctly performed and that a certain percentage of failed manoeuvres will occur, despite training and experience. The higher number of acceptable manoeuvres in the present study might be a result of more frequent measurements (i.e. three times per week).

The same consistent result was not found for inspiratory capacity (IC), a lung function measure that may indirectly reflect the severity of lung hyperinflation, which represents the fundamental origin of dyspnoea development in COPD. IC has thus been suggested to better reflect dyspnoea than $\mathrm{FEV}_{1}$ [32] and to be a stronger predictor of exacerbations when divided by the total lung capacity of the patient [33]. Therefore, IC was chosen as a routine measurement in the present study. However, this manoeuvre was found to be harder to perform correctly unsupervised compared to $\mathrm{FEV}_{1}$. Consequently, it was more difficult to interpret data from the IC-manoeuvres, making this parameter less suitable to measure in an unsupervised procedure. This might reflect that COPD patients in general are much more used to perform the $\mathrm{FEV}_{1}$ manoeuvres, even though they are more effort dependent. We still believe that IC might be a promising candidate for telehealth monitoring purposes as a few of the participants in the study showed a correlation between IC and symptoms, a correlation less prominent for $\mathrm{FEV}_{1}$ (Table 4).

\section{Clinical implications}

Telehealth is considered to enable close monitoring of the patient in a real life situation as well as transferring data on a regular basis to the clinic. Home monitoring of patients with COPD has received increased attention the last years. Both to help patients with self-management strategies but also to be able to detect exacerbations and by preset alarms help decision making for healthcare professionals. Many telehealth systems have been designed to identify signs of COPD exacerbations, but few previous studies have reported the nature of recorded lung function data and what variations to expect in stable versus unstable patients. This is of relevance when interpreting results of home monitored lung function parameters. From a clinical perspective, it is also important to relate the variation in lung function to the spirometric COPD grade. However, this could not be done in this study due to the low number of participants.

\section{Study limitations}

In this study, we used a pragmatic approach. The patients got limited support which may explain especially the results of the IC-measurements. Using some kind of guidance or feedback, the IC-manoeuvres might have been performed more like in clinic. It could easily be performed by including an instruction video as well as visual or audio feedback on the spirometry manoeuvre. This is in line with the recent review by Baroi et al. who concluded that remote respiratory assessments are feasible when combined with sufficient organizational back up [27]. However, considering the lack of feedback, we are surprised that the patients performed acceptable manoeuvres to such a large extent.

In the design of the study we aimed to recruit patients with frequent exacerbations. The majority of patients had a history of two or more exacerbations the previous year before inclusion. Though, the lack of exacerbation episodes during the study period makes it impossible to identify characteristics of individuals who might benefit the most of such a telehealth system at home and further studies are warranted.

\section{Conclusions}

The study presents a large, intra-individual variation in lung function during the total of 240 patient weeks of monitoring. The variation was present even though neither exacerbations nor corresponding changes in symptoms occurred. This is important to take into consideration when monitoring patients at home in order to detect exacerbations. The multi-item telehealth system for home monitoring was feasible to employ in a home environment for patients with COPD, since more than $98 \%$ of the planned manoeuvres were conducted with acceptable within-manoeuvre variability. We also conclude that the IC-manoeuvres seemed to be more difficult than the forced expiratory manoeuvres to perform unsupervised. 


\section{Supplementary information}

Supplementary information accompanies this paper at https://doi.org/10. 1186/s12911-020-1103-6.

Additional file 1: Supplementary Materials. Lung function measurements.

\begin{abstract}
Abbreviations
AE: Acute exacerbations; AM: Morning; ATS: American Thoracic Society; CAT: COPD assessment test; COPD: Chronic obstructive pulmonary disease; CV: Coefficient of variation; ERS: European Respiratory Society; FEV ${ }_{1}$ : Forced expiratory volume in one second; $F_{E V}$ : Forced expiratory volume in six seconds; FVC: Forced vital capacity; GOLD: Global initiative for chronic Obstructive lung disease; IC: Inspiratory capacity; IQR: Inter Quartile Range; mMRC: Modified Medical Research Council Dyspnea scale; PM: Afternoon/ evening; $\mathrm{SpO}_{2}$ : Oxygen saturation; $\mathrm{TH}$ : Telehealth
\end{abstract}

\section{Acknowledgements}

We truly acknowledge the amount of time all the study participants put into this study.

\section{Authors' contributions}

ÅH: Creation of question, responsible for designing the TH system, taking part in data collection and data analysis, writing and reviewing manuscript, reviewing tables and figures. FÖ: Taking part in designing the TH system and data collection, responsible for the statistical analysis, writing and reviewing manuscript. UW: Performed analyses of recordings, responsible for the figures and tables, writing and reviewing manuscript. EB: Responsible for data collection and reviewing manuscript. AB: Creation of question, writing and reviewing manuscript, reviewing tables and Figs. KW: Creation of question, taking part in the data collection and analysis, writing and reviewing manuscript, reviewing tables and Figs. KW is the senior author and guarantor of this publication. The author(s) read and approved the final manuscript.

\section{Funding}

The study received funding from the Swedish Research Council (K2014-99X22572-01-4). Open access funding provided by Umea University

\section{Availability of data and materials}

The datasets used and/or analysed during the current study are available from the corresponding author on reasonable request.

\section{Ethics approval and consent to participate}

The Regional ethical review board at Umeå University accepted this study; Registration number: 2013-143-32M (complement to 2011-9-31M). Each participant gave his/her written informed consent before entering the study.

\section{Consent for publication}

The participants did not sign a specific consent for publication. The plan for publication was included in the ethical application. Information about the participants' confidentiality was included in the consent to participate.

\section{Competing interests}

The authors declare that they have no competing interests.

\section{Author details}

'Department of Radiation Sciences, Biomedical Engineering, Umeå University, Umeå, Sweden. ${ }^{2}$ Department of Epidemiology and Global Health, Umeå University, Umeå, Sweden. ${ }^{3}$ Department of Public Health and Clinical Medicine, Section of Medicine, Umeå University, Umeå, Sweden. ${ }^{4}$ Department of Community Medicine and Rehabilitation, Physiotherapy, Umeå University, Umeå, Sweden.

Received: 19 November 2019 Accepted: 27 April 2020 Published online: 12 May 2020

\section{References}

1. Lozano R, Naghavi M, Foreman K, Lim S, Shibuya K, Aboyans V, et al. Global and regional mortality from 235 causes of death for 20 age groups in 1990 and 2010: a systematic analysis for the global burden of disease study 2010. Lancet. 2012;380(9859):2095-128.

2. Vos T, Flaxman AD, Naghavi M, Lozano R, Michaud C, Ezzati M, et al. Years lived with disability (YLDs) for 1160 sequelae of 289 diseases and injuries 1990-2010: a systematic analysis for the global burden of disease study 2010. Lancet. 2012;380(9859):2163-96.

3. Vogelmeier CF, Criner GJ, Martinez FJ, Anzueto A, Barnes PJ, Bourbeau J, et al. Global Strategy for the Diagnosis, Management, and Prevention of Chronic Obstructive Lung Disease 2017 Report: GOLD executive summary. Eur Respir J. 2017;49(3).

4. Soler-Cataluna JJ, Martinez-Garcia MA, Roman Sanchez P, Salcedo E, Navarro M, Ochando R. Severe acute exacerbations and mortality in patients with chronic obstructive pulmonary disease. Thorax. 2005;60(11):925-31.

5. Seemungal TA, Wedzicha JA. Acute exacerbations of COPD: the challenge is early treatment. Copd. 2009;6(2):79-81.

6. Baroi S, McNamara RJ, McKenzie DK, Gandevia S, Brodie MA. Advances in remote respiratory assessments for people with chronic obstructive pulmonary disease: a systematic review. Telemed J E Health. 2017.

7. Mohktar MS, Redmond SJ, Antoniades NC, Rochford PD, Pretto JJ, Basilakis J, et al. Predicting the risk of exacerbation in patients with chronic obstructive pulmonary disease using home telehealth measurement data. Artif Intell Med. 2015;63(1):51-9.

8. Sund ZM, Powell T, Greenwood R, Jarad NA. Remote daily real-time monitoring in patients with COPD --a feasibility study using a novel device. Respir Med. 2009;103(9):1320-8.

9. Segrelles Calvo G, Gomez-Suarez C, Soriano JB, Zamora E, Gonzalez-Gamarra A, Gonzalez-Bejar M, et al. A home telehealth program for patients with severe COPD: the PROMETE study. Respir Med. 2014;108(3):453-62.

10. Cordova FC, Ciccolella D, Grabianowski C, Gaughan J, Brennan K, Goldstein $F$, et al. A telemedicine-based intervention reduces the frequency and severity of COPD exacerbation symptoms: a randomized, controlled trial. Telemed J E Health. 2015.

11. MacNab M, Lee SH, McCloughan L, Hanley J, McKinstry B, Pinnock H. Oximetrysupported self-management for chronic obstructive pulmonary disease: mixed method feasibility pilot project. BMC Health Serv Res. 2015;15:485.

12. Tupper OD, Gregersen TL, Ringbaek T, Brondum E, Frausing E, Green A, et al. Effect of tele-health care on quality of life in patients with severe COPD: a randomized clinical trial. Int J Chron Obstruct Pulmon Dis. 2018;13: 2657-62.

13. Sanchez-Morillo D, Fernandez-Granero MA, Jimenez AL. Detecting COPD exacerbations early using daily telemonitoring of symptoms and k-means clustering: a pilot study. Med Biol Eng Comput. 2015;53(5):441-51.

14. Al Rajeh AM, Hurst JR. Monitoring of Physiological Parameters to Predict Exacerbations of Chronic Obstructive Pulmonary Disease (COPD): A Systematic Review. J Clin Med. 2016;5(12).

15. Garcia-Sidro P, Naval E, Martinez Rivera C, Bonnin-Vilaplana M, Garcia-Rivero $J$, Herrejon A, et al. The CAT (COPD assessment test) questionnaire as a predictor of the evolution of severe COPD exacerbations. Respir Med. 2015; 109(12):1546-52.

16. Steventon A, Ariti C, Fisher E, Bardsley M. Effect of telehealth on hospital utilisation and mortality in routine clinical practice: a matched control cohort study in an early adopter site. BMJ Open. 2016;6(2):e009221.

17. Ringbaek T, Green A, Laursen LC, Frausing E, Brondum E, Ulrik CS. Effect of tele health care on exacerbations and hospital admissions in patients with chronic obstructive pulmonary disease: a randomized clinical trial. Int J Chron Obstruct Pulmon Dis. 2015;10:1801-8.

18. Hamad GA, Crooks M, Morice AH. The value of telehealth in the early detection of chronic obstructive pulmonary disease exacerbations: a prospective observational study. Health Informatics J. 2016;22(2):406-13.

19. Pedone C, Chiurco D, Scarlata S, Incalzi RA. Efficacy of multiparametric telemonitoring on respiratory outcomes in elderly people with COPD: a randomized controlled trial. BMC Health Serv Res. 2013;13:82.

20. Kruse C, Pesek B, Anderson M, Brennan K, Comfort H. Telemonitoring to manage chronic obstructive pulmonary disease: systematic literature review. JMIR Med Inform. 2019;7(1):e11496.

21. Ohberg F, Wadell K, Blomberg A, Claesson K, Edström U. A H. home-based system for recording pulmonary function and disease-related symptoms in patients with chronic obstructive pulmonary disease, COPD - a pilot study. SM J Pulm Med. 2016;2(1):1011-6.

22. Jones PW. COPD assessment test --rationale, development, validation and performance. Copd. 2013;10(2):269-71. 
23. Holland AE, Spruit MA, Troosters T, Puhan MA, Pepin V, Saey D, et al. An official European Respiratory Society/American Thoracic Society technical standard: field walking tests in chronic respiratory disease. Eur Respir J. 2014; 44(6):1428-46.

24. Mahler DA, Wells CK. Evaluation of clinical methods for rating dyspnea. Chest. 1988;93(3):580-6.

25. Miller MR, Hankinson J, Brusasco V, Burgos F, Casaburi R, Coates A, et al. Standardisation of spirometry. Eur Respir J. 2005;26(2):319-38.

26. Pellegrino R, Rodarte $J R$, Brusasco V. Assessing the reversibility of airway obstruction. Chest. 1998;114(6):1607-12.

27. Baroi S, McNamara RJ, McKenzie DK, Gandevia S, Brodie MA. Advances in remote respiratory assessments for people with chronic obstructive pulmonary disease: a systematic review. Telemed J E Health. 2018;24(6):415-24.

28. Rodriguez-Roisin R, Tetzlaff $\mathrm{K}$, Watz $\mathrm{H}$, Wouters EF, Disse B, Finnigan $\mathrm{H}$, et al. Daily home-based spirometry during withdrawal of inhaled corticosteroid in severe to very severe chronic obstructive pulmonary disease. Int J Chron Obstruct Pulmon Dis. 2016;11:1973-81.

29. Janssens W, Liu Y, Liu D, Kesten S, Tashkin DP, Celli BR, et al. Quality and reproducibility of spirometry in COPD patients in a randomized trial (UPLIFT(R)). Respir Med. 2013;107(9):1409-16.

30. Cazzola M, MacNee W, Martinez FJ, Rabe KF, Franciosi LG, Barnes PJ, et al. Outcomes for COPD pharmacological trials: from lung function to biomarkers. Eur Respir J. 2008;31(2):416-69.

31. Redelmeier DA, Bayoumi AM, Goldstein RS, Guyatt GH. Interpreting smal differences in functional status: the six minute walk test in chronic lung disease patients. Am J Respir Crit Care Med. 1997:155(4):1278-82.

32. Cui L, Ji X, Xie M, Dou S, Wang W, Xiao W. Role of inspiratory capacity on dyspnea evaluation in COPD with or without emphysematous lesions: a pilot study. Int J Chron Obstruct Pulmon Dis. 2017;12:2823-30.

33. Zaman M, Mahmood S, Altayeh A. Low inspiratory capacity to total lung capacity ratio is a risk factor for chronic obstructive pulmonary disease exacerbation. Am J Med Sci. 2010;339(5):411-4.

\section{Publisher's Note}

Springer Nature remains neutral with regard to jurisdictional claims in published maps and institutional affiliations.

Ready to submit your research? Choose BMC and benefit from:

- fast, convenient online submission

- thorough peer review by experienced researchers in your field

- rapid publication on acceptance

- support for research data, including large and complex data types

- gold Open Access which fosters wider collaboration and increased citations

- maximum visibility for your research: over $100 \mathrm{M}$ website views per year

At $\mathrm{BMC}$, research is always in progress.

Learn more biomedcentral.com/submissions 